\title{
La relación entre el nivel pobreza, la tasa de analfabetismo y el ingreso per cápita en Honduras, 2006-2011
}

\author{
César Yanuario Hernández C. \\ Joseph Malta ${ }^{2}$
}

\section{RESUMEN}

El presente artículo muestra los resultados del análisis de la relación existente entre los niveles de pobreza, la tasa de analfabetismo y el ingreso per cápita a nivel nacional, para el período 2006-2011. Los productos fueron obtenidos a partir de la utilización de la metodología de las bases de estadística de la encuesta permanente de hogares de propósitos múltiples (EPHPM-Mayo).

Se resalta la tendencia de la pobreza, el analfabetismo y el ingreso per cápita; lo importante en todo esto son los comportamientos que han venido mostrando estos aspectos y la explicación del fenómeno de la pobreza. Así, se deduce de forma general, que la pobreza en Honduras está vinculada al tema de educación e ingreso. Además, existen otros aspectos que la determinan, que para propósitos de esta investigación se ha reducido a la tasa de analfabetismo y al ingreso per cápita.

No obstante, esto no quiere decir que estos parámetros sean los más apropiados para explicar de manera precisa la pobreza, ya que por naturaleza es un tema complejo de analizar. En esencia, lo que se busca es mostrar el comportamiento de la pobreza en nuestro país y su relación con la educación y el ingreso.

Palabras clave: pobreza, pobreza relativa, pobreza extrema, tasa de analfabetismo, ingreso percápita.

\section{ABSTRACT}

The follow article shows the analysis results of the relationship between the poverty levels, literacy rate, and the income per capita nationwide, for the period 2006-2011. These results were obtained of the use of the methodology centered in the statistic basis of the Encuesta Permanente de Hogares de Propositos Multiples (EPHPM-

\footnotetext{
${ }^{1}$ Beneficiario de una beca básica de la DICYP. Estudiante de la Maestría en Metodologías de Investigación Económica y Social, Facultad de Ciencias Económicas, UNAH: cesaryanuario@hotmail.com

${ }^{2}$ Asesor, coordinador de área, Vicerrectoría Académica.
} 
Mayo). It highlights the tendency of the poverty, literacy, and the income per capita; the important from this, are the behaviors that have been showing these aspects and the meaning of the poverty phenomenon. Generally follows that poverty in Honduras is linked with the issue of education and the income. Besides, there are other aspects that determine it; in view of the issues raised in this article, it has reduced to the literacy rate and the income per capita. It does not want to say, these parameters are the most appropriate way for explaining poverty, for naturally is a complex issue of analyzing. In essence what is sought is to show the behavior poverty in our country and his relationship between education and the income.

Keywords: poverty, relative poverty, extreme poverty, illiteracy rate, income per capita.

\section{INTRODUCCIÓN}

La elección del tema de estudio tiene por propósito la revisión del panorama socioeconómico, dentro del cual la pobreza es uno de los problemas que más afecta a la población de hondureña, puesto que se considera como un fenómeno relacionado a la carencia de las condiciones humanas dignas.

Según la Estrategia para la Reducción de la Pobreza (ERP), la pobreza es una condición económica y social con causas y expresiones múltiples, pero la manera más común y simple de presentarla es como una insuficiencia de ingresos para alcanzar un cierto nivel mínimo de vida; además de que su concepto comprende el grado de insatisfacción acerca de un conjunto de necesidades humanas básicas. Así, al analizar en forma comparativa las características socioeconómicas de la sociedad hondureña, se observa que la pobreza ha afectado drásticamente a una buena parte de la población en esta condición.

El artículo se realizó con información obtenida de la base de datos de la encuesta permanente de hogares de propósitos múltiples, la cual es presentada al mes de mayo en cada año. Se determinó el nivel y las características socioeconómicas de la población considerada pobre, utilizando el método de medición, conocido como línea de pobreza, en donde los pobres son aquellos que viven en hogares cuyo ingreso por persona es inferior al costo de una canasta básica de consumo.

También se enfatiza el análisis de la relación entre el nivel de pobreza, la tasa de analfabetismo y la determinación del ingreso per cápita, describiendo tendencias y considerando el panorama socioeconómico de la población urbano-rural con el propósito de lograr una aproximación en materia de desarrollo humano en Honduras. Asimismo, se planteó estructurar el artículo en cuatro apartados. 
El primero contiene los aspectos teóricos de la pobreza, tasa de analfabetismo, el ingreso per cápita a nivel nacional y el índice de Gini como indicador de la distribución del ingreso; el segundo, describe la tendencia de la pobreza relativa y extrema en la área urbana-rural, reflejando la brecha entre ambos tipos de pobreza, de forma similar se presenta un panorama de la población en condición de pobreza; en el tercero se propone una descripción de la tasa de analfabetismo en la población mayor de 15 años a nivel nacional, del ingreso per cápita y del coeficiente o índice de Gini, mostrando la tendencia de la tasa de analfabetismo entre dominios urbanorural y el ingreso per cápita generado a nivel nacional, resaltando también el ingreso per cápita en comparación con los años de estudio promedio, así como un comparativo de ingreso entre género; y, por último, se muestra la tendencia del índice de Gini.

\section{MÉTODOLOGÍA}

La metodología utilizada en esta investigación se basa en los principios básicos de la estadística descriptiva, con el propósito de caracterizar series y tendencias de la pobreza. Tales herramientas aportan un análisis de regresión econométrico en donde se plantea un modelo logarítmico; la variable explica la pobreza, la cual está en función de las variables explicativas: tasa de analfabetismo y el coeficiente de Gini, en semejanza al ingreso per cápita, para ello se usaron técnicas econométricas aplicadas con el programa econométrico STATA.

Para poder modelar, se creó una base de serie tiempo del año 2001 al 2011, de esta forma se potenció el análisis econométrico. En cuanto a la parte descriptiva, se utilizaron las herramientas del programa EXCEL, generando la mayor parte del análisis con gráficos y cuadros. El artículo cuenta con un diseño de investigación cuantitativo con enfoque descriptivo; asimismo, las bases de datos se fragmentaron para facilitar el análisis, tal como pobreza relativa, extrema, tasa de analfabetismo e ingreso per cápita.

\section{A. Aspectos teóricos de la pobreza, tasa de analfabetismo y el ingreso per cápita}

\section{Lapobreza en Honduras}

La pobreza se conoce como: "Un síndrome situacional en el que se asocia el infraconsumo, la desnutrición, las precarias condiciones de las viviendas, los bajos niveles de educación, las malas condiciones sanitarias, una inserción inestable en el aparato productivo o dentro de los estratos primitivos del mismo, actitudes de desaliento y anomalía, poca participación en los 
mecanismos de integración social y quizás las descripción a una escala particular de valores, diferenciada en alguna manera de la del resto de la sociedad" (Altimir,1970). Tal planteamiento de Altimir define el concepto de pobreza de forma amplia y precisa, aunque debería agregar algunos aspectos que son considerados en la actualidad, al estar asociados a la situación de pobreza, porque principalmente se asocia el problema al infraconsumo; de esta forma, Townswend la define así: "Los individuos, familias y grupos de población se consideran pobres cuando carecen de los recursos necesarios para obtener el tipo de dietas, participar en actividades y tener las condiciones de vida y equipamiento que se acostumbran o, por lo menos, las que son ampliamente promovidas y aprobadas en las sociedades a las que pertenecen."

La pobreza es una condición indignante para el ser humano, porque desnaturaliza al humano y lo reduce a desecho de la sociedad, por eso este flagelo es uno de los grandes problemas que agobian a la población en el país. Hace muchos años la pobreza ha generado un sinnúmero de problemas, según algunos expertos esta tiene relación con los niveles de inseguridad y violencia. En síntesis, la pobreza se debe al estado de precariedad humana, tal como se puede dilucidar de las anteriores definiciones.

El método que se utiliza para medir la pobreza en Honduras, línea de la pobreza, consiste en establecer, a partir de los ingresos de los hogares, la capacidad que estos tienen para satisfacer, por medio de la compra de bienes y servicios, un conjunto de necesidades alimentarias y no alimentarias consideradas como básicas.

El enfoque más importante de percibir la pobreza es, por el lado de los ingresos, donde se percibe a la pobreza como una falta de ingresos ( 0 consumo) que no permite necesidades básicas. Estas necesidades básicas pueden estar definidas por un concepto absoluto o relativo; por eso el enfoque multidimensional considera que la falta de ingresos no es la única clase de privación que las personas pueden sufrir; se trata de incluir otras dimensiones al nivel y la calidad de vida, en esto se incluyen la educación, nutrición, inseguridad, vulnerabilidad, acceso a servicios básicos.

El enfoque de las necesidades básicas insatisfechas (NBI) se define como un conjunto taxativo de necesidades que determinan si un hogar o persona es pobre, dentro de ellas se mencionan el hacimiento, características de la vivienda, abastecimiento de agua, acceso a saneamiento, asistencia a 
escuelas primarias de los menores en edad escolar y algún indicador indirecto de capacidad económica.

El enfoque de las capacidades se refiere a que cuanto mayor es el ingreso de una persona, mayor es su control sobre los productos básicos y, por lo tanto, aumentan los medios para tener una vida mejor; lo que quiere decir que el ingreso es un medio para lograr un fin. Para Amartya Sen, lo importante es lo que se puede o no hacer, lo que se puede o no ser; por lo tanto, el nivel de vida que las personas disfrutan debe ser visto en función de los logros individuales que son posibles y no en función de los medios que las personas poseen para lograrlos. Sen desarrolló los conceptos de funcionamientos y capacidades, donde un funcionamiento es un logro y una capacidad para lograr algo; los funcionamientos están directamente relacionados con el tipo de vida (el estándar de vida) que las personas tienen y las capacidades son las oportunidades con las que cuentas las personas para tener la vida de su elección.

\section{Analfabetismo}

El analfabetismo es un problema que impacta de manera muy importante en la realidad educativa del país. En realidad, se plantea como un problema de carácter social y, consecuentemente, de naturaleza política, tal como lo ha mostrado la concepción "freiriana" al entenderlo no como problema político, sino como de carácter moral (Jiménez Castillo, 2007).

La incapacidad de poder leer y escribir es otro de los problemas graves que afronta el país, este es un proceso mediante el cual se transmiten conocimientos y, por ende, tiene un papel fundamental en la sociedad. Tales competencias les permiten a las personas ser más desarrolladas, pues las poblaciones que han mejorado los niveles educativos poseen capacidades y esto convierte a una sociedad en competitiva, productiva y sobre todo consiente de la realidad de su entorno. Básicamente, es lo que se esperaría en esta dinámica de la formación profesional, porque su impacto se considera un factor que determina una mayor remuneración personal.

Algunos datos para el año 2006 reflejan que la tasa de analfabetismo en Honduras llegó al $17.5 \%$, para el año 2011 presentó una tasa del $14.5 \%$; con lo cual se puede observar que en este periodo de 6 años la tasa ha venido disminuyendo, lo que se puede explicar por los avances en los planes estratégicos que ha promocionado el Secretaría de Educación en esta materia. 
3. Ingreso per cápita

El ingreso per cápita es la relación que hay entre el producto interno bruto (PIB) y la cantidad de habitantes de un país. Para obtenerlo se divide el PIB entre su población.

Es un indicador que permite medir el crecimiento económico de una nación, en vista que ofrece una noción adecuada de los patrones de calidad de vida de la población dentro de un país, en términos de bienestar económico. Se estima como el ingreso total del hogar dividido entre los miembros del hogar (INE). Es un indicador comúnmente usado para estimar la riqueza económica de un país, ya que numerosas evidencias muestran que la renta per cápita está positivamente correlacionada con la calidad de vida de los habitantes de un país.

4. El índice de Gini

Es uno de los indicadores más utilizados para medir la desigualdad en la población de un determinado país. Este se deriva directamente de la curva de Lorenz, definida como un instrumento gráfico que permite medir la desigualdad, para dibujar los receptores del ingreso que son ordenados de menor a mayor, midiéndose en el eje horizontal el porcentaje de personas según el nivel de ingreso; en el eje vertical se mide el porcentaje de ingresos recibidos por cada grupo de la población.

De este modo, un punto de la curva de Lorenz indica qué porcentaje del total de ingresos es recibido por un porcentaje específico de la población. Normalmente, se utiliza para medir la desigualdad en los ingresos dentro de un país, pero puede utilizarse para medir cualquier forma de distribución desigual.

Como ya se dijo, el índice de Gini se deriva de la curva de Lorenz, es el valor del área $M$ dividido entre el área $(M+N)$. A mayor $M$, mayor desigualdad; es decir, que mientras más cercano sea el índice a la unidad, existirá un mayor grado de desigualdad.

Amartya Sen define este índice de la siguiente manera: "La función de bienestar implícita (que es medida por el índice de Gini), es una suma de las porciones del ingreso de varias personas ponderadas por el lugar ocupado en el ordenamiento". 
Figura 1. Curva de Lorenze índice de Gini

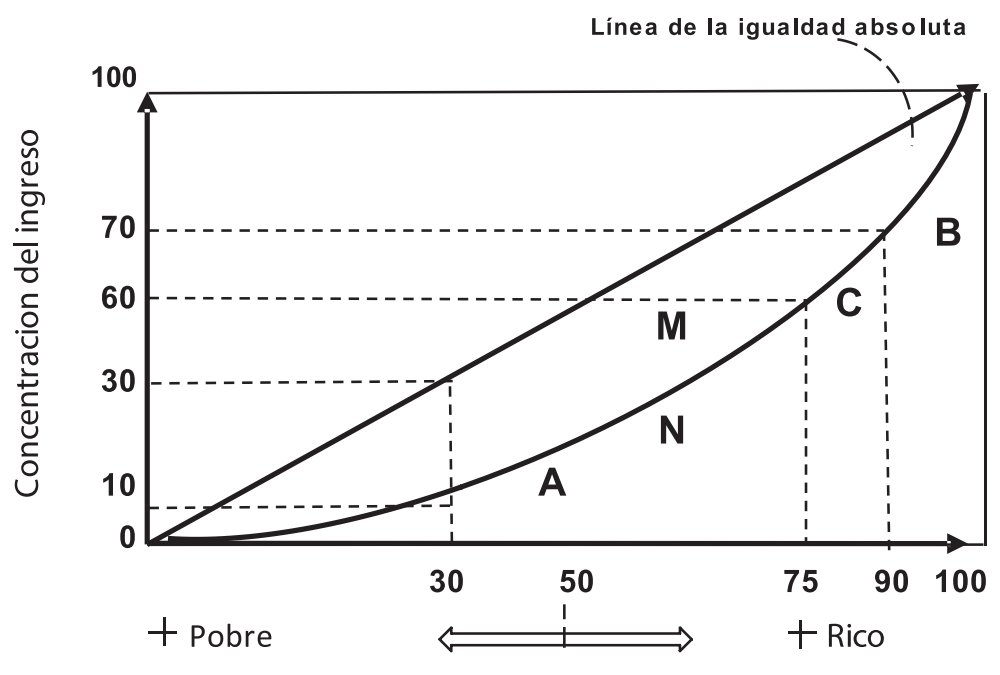

Concentración de la población

B. Caracterización de la incidencia de la pobreza por personas que viven en hogares por nivel de pobreza

La pobreza es un problema generalizado que afecta a la mayoría de la población del país, las características que normalmente se presentan en condiciones de pobreza son lo falta de salud, de vivienda, educación, ingresos empleo, etc. En resumen, es la carencia de recursos desde el punto de vista económico.

En gran medida se puede decir que las características de la población en condición de pobreza están determinadas por los niveles de educación y de ingresos, enfocados en la incidencia que estas variables tienen en el nivel de pobreza.

La población total nacional de Honduras para el año 2011 presentó un total de 8, 200,795 habitantes; de los cuales 3, 987,236 son hombres y 4, 213, 559 son mujeres. La población económicamente activa asciende en total a nivel nacional, para el año 2011, a 3, 369, 919; de los cuales 2, 183, 811 son hombres y 1, 186, 107 son mujeres. El ingreso per cápita de los hogares para este mismo año fue de USD 2, 774 .

La población total a nivel nacional que se encuentra en pobreza, tanto relativa como extrema para el año 2011, ascendió a 5,493, 390; de los cuales 1, 683, 036 están en pobreza relativa y $3,810,354$ en pobreza extrema; lo que en términos 
porcentuales a nivel nacional quiere decir que la pobreza ascendió a una tasa del $67.6 \%$ y para la pobreza relativa y extrema al $20.7 \%$ y $46.9 \%$, respectivamente.

Este es el escenario que se tiene en el país, situación definitivamente alarmante desde todo punto de vista; lo cual se tratará de describir de forma precisa en los siguientes apartados.

\section{Tendencia de la pobreza total en Honduras, período 2006-2011}

Al analizar la pobreza total en Honduras durante el período 2006-2011, se observa un panorama de la realidad del país en donde la tendencia muestra un crecimiento acelerado durante este período de estudio. A inicios de 2006, la pobreza presentó una tasa del $60 \%$ y para el año 2011 mostró un crecimiento significativo del $67 \%$ (ver gráfico 1), mostrando una reducción leve en los años 2007 y 2009.

Lo anterior significa que los pobres para el año 2006 eran 4, 395,837 y 5, 493,390 para el año 2011; por diferencia simple se ve que en este corto período de estudio de 6 años, la pobreza total a nivel nacional se incrementó en $1,097,553$ nuevos pobres.

De esta forma, es posible señalar el incremento del nivel de pobreza en el país, lo que se deriva de muchas razones directamente relacionadas con la política del Gobierno, pero que en este estudio se ha limitado solamente a la importancia que tiene la educación y el ingreso en la sociedad.

En este sentido, se puede deducir que sin medidas eficaces y carentes de seriedad, la tendencia de la pobreza total a nivel nacional será hacia el crecimiento para los próximos años, de modo que sino se implementan medidas que mejoren la economía nacional y, por ende, los ingresos de la población, el efecto será el mismo.

El gráfico 1 muestra la tendencia de la pobreza en este período de estudio, en este se observa que para el año 2006 la tasa de pobreza fue del $62 \%$ y para el año 2011 ascendió al 67 \%; en este período solamente en los años 2007 y 2009 se presentó una leve reducción de la tasa de pobreza. En estos resultados se aprecia que la tasa de pobreza se incrementó en 5 puntos porcentuales en un período de 6 años, lo cual permite deducir que la tendencia para los próximos años de la tasa de pobreza en el país es hacia un crecimiento. 
Gráfico 1. Pobreza total, 2006-2011

$\multimap$ Tasa de Pobreza

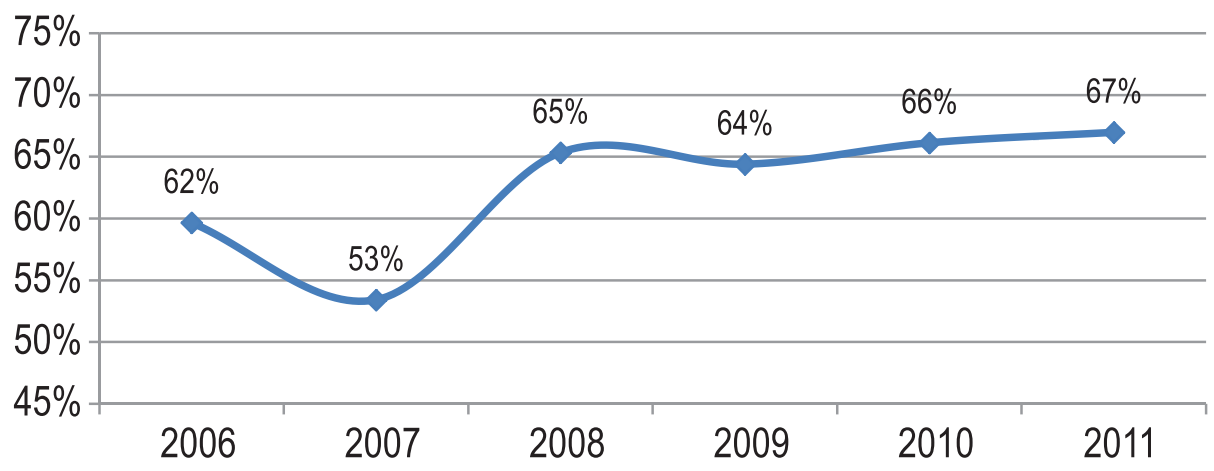

Fuente: elaboración propia con base a INE, EPHPM; a mayo de 2006-2011.

2. Tendencia de la pobreza total, relativa y extrema en Honduras

En el período de estudio se observa que la pobreza se redujo durante el 2007, especialmente la pobreza extrema, la cual registra una reducción del $19.2 \%$, no obstante, a partir de 2008 siguió mostrando una tendencia de crecimiento de forma leve; no así la pobreza relativa, que presentó una inclinación igual, pero se redujo para los años 2010 y 2011.

Lo que significa que en el caso de la pobreza extrema, para el año 2006 era de $3,139,792$ pobres extremos y para el año 2007 se redujo a 2,537,117; lo que sería una reducción de 602, 675 pobres en condición extrema.

Al comparar la pobreza relativa y extrema, se puede observar que la primera es en donde la mayor cantidad de personas se aglomeran, o sea, que el problema de la pobreza se manifiesta de forma profunda en este sector de la población, la cual sufre de forma trágica las condiciones humanas más precaria del país. Tal como se aprecia en el gráfico 2, para el año 2011 la población en pobreza extrema reflejó la cantidad de $3,810,354$ personas y para la pobreza relativa 1 , 683,036 ; datos que revelan una marcada brecha entre ambos sectores de la población en esta condición.

Lo anterior se traduce en que la pobreza extrema en este período de estudio se incrementó de mayor forma que la pobreza relativa; lo cual significa que la tendencia de crecimiento mayor entre estas dos pobrezas es en la pobreza extrema. 
Gráfico 2. Pobreza relativa extrema, 2006-2011

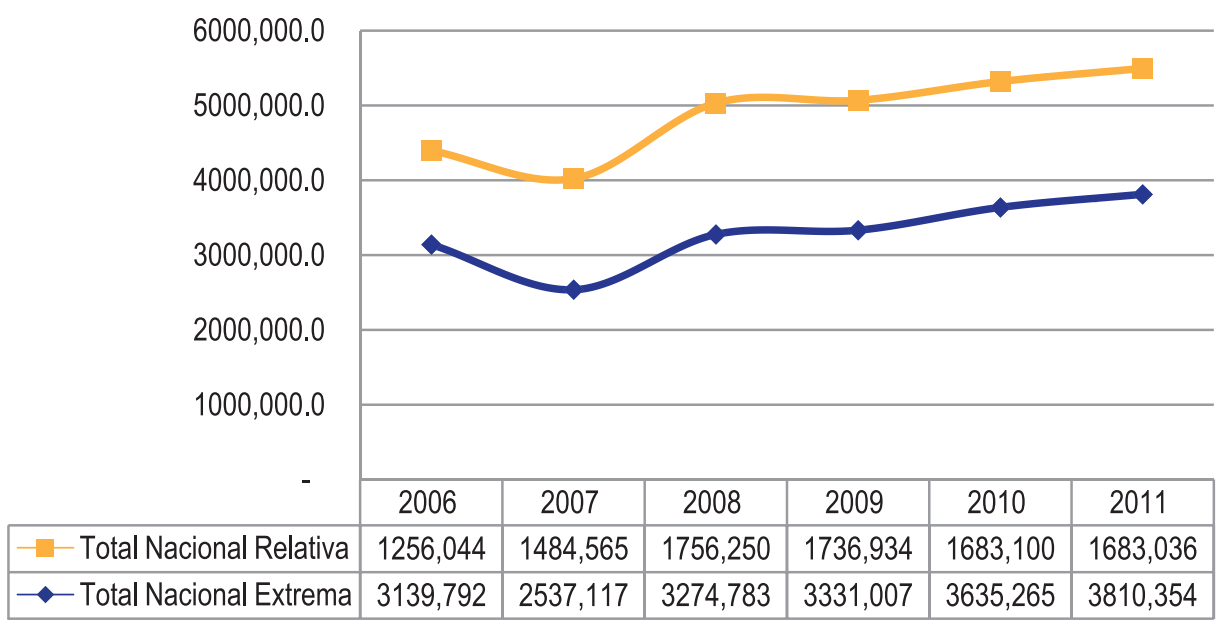

Fuente: elaboración propia con base a INE, EPHPM, mayo de 2006-2011.

El gráfico 2 muestra una tendencia hacia el crecimiento de la pobreza; para el año 2006, la pobreza relativa y extrema fue de un $19.3 \%$ y $42.5 \%$, respectivamente. Lo importante de resaltar aquí es que la brecha se ha venido marcando entre ambas: pobreza relativa y extrema. En el año 2006, la brecha fue de 1, 883,748 y prácticamente para el año 2011 la diferencia fue 2, 127,318 ; lo que significa que la brecha entre pobreza relativa y extrema se incrementó en este período de estudio.

3. Tendencia de la pobreza total en las áreas urbano-rural

En lo que respecta a la pobreza en las áreas urbano-rural, se destaca que la pobreza se ha venido concentrando en sectores rurales. El análisis de los datos de la encuesta de hogares de propósitos múltiples del Instituto Nacional de Estadísticas de Honduras (INE, 2001-2010), refleja hallazgos importantes, los cuales se resumen a continuación en forma detallada.

A nivel nacional, la pobreza ha mostrado una brecha entre la pobreza radicada en los dominios urbano y rural, muestra de que la situación es más complicada en el área rural del país. La pobreza para el período de estudio se estima que ha tenido un crecimiento promedio del $4.56 \%$; lo que se puede explicar que por cada año en promedio ingresan en condición de pobreza 219,511 personas. Tales estimaciones indican que el fenómeno de la pobreza continua siendo mayor en la población del área rural con 2516,507 personas; 
frente a 1, 879,330 de personas en el área urbana. La tendencia muestra que la pobreza total se redujo en el año 2007, sobre todo el área rural, que para el mismo año presenta una disminución del $16.7 \%$. Posteriormente, es posible afirmar que la tendencia de la pobreza presenta un leve estancamiento, pero de igual forma creciente.

Los niveles de pobreza son más elevadas en el área rural que el área urbana. De acuerdo al IDH (2011), esto se debe a que la primera presenta grandes limitaciones en cuanto a cobertura y calidad de los servicios sociales, menores oportunidades laborales y en ella predomina una economía de subsistencia, principalmente agrícola, con una reducida interacción con los mercados, con trabas estructurales y socioeconómicas para acceder al crédito, menos competitiva y con menores opciones de diversificación (ver gráfico 3).

Gráfico 3. Pobreza total urbana-rural, 2006-2011

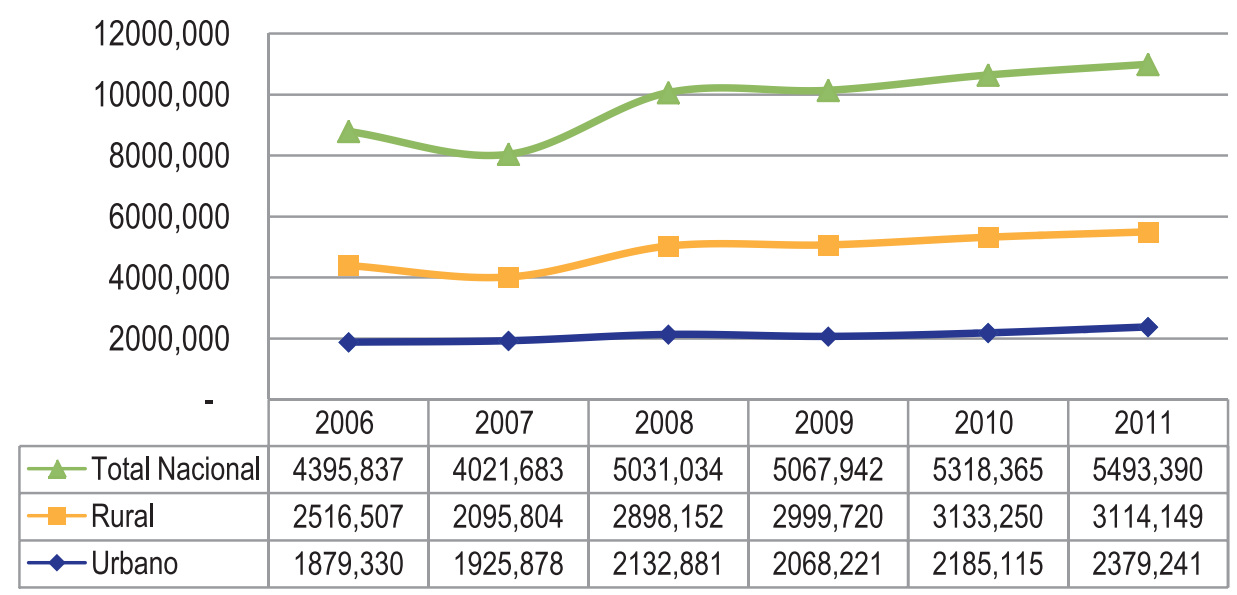

Fuente: elaboración propia con base a INE, EPHPM, mayo de 2006-2011.

El análisis de la pobreza en Honduras según el IDH (2011), señala que el país necesita una estrategia para la reducción de la pobreza que sea integral, diferenciada y focalizada, es decir, retomar la implementación de la ERP. Este Informe hace énfasis en que en el país es importante retomar la ERP para poder alcanzar avances significativos con miras a la disminución de la pobreza. Hasta ahora, no es cierto que se haya avanzado en la solución a este problema; por lo tanto, es necesaria la actualización y revisión de esta problemática, pero bajo un enfoque integral, diferenciado y focalizado, tal como lo plantea el IDH (2011) para la incorporación y ejecución de la estrategia de la reducción de la pobreza en la agenda nacional. 
C. Caracterización de la tasa de analfabetismo, el ingreso per cápita y el coeficiente e índice de Gini

A continuación se describe el comportamiento de la tasa de analfabetismo, el ingreso per cápita e índice de Gini y se realiza un breve análisis descriptivo de las tendencias de cada uno de los tres aspectos anteriores, basándose en un análisis econométrico.

1. Tendencia de la tasa de analfabetismo, según dominios en el período 20062011

De acuerdo con la EPHPM, la tendencia que ha venido mostrando el analfabetismo es otro de los grandes problemas de relevancia en el país, sobre todo en sectores de la población que muestran bajos niveles de educación.

Según estimaciones de la misma fuente en lo que corresponde a la tasa de analfabetismo a nivel nacional, son las personas mayores de 15 años las que nos saben leer ni escribir; lo cual indica que a nivel nacional, para el año 2006, este indicador fue de un $17.5 \%$, disminuyendo de forma leve para el año 2011 a un $14.9 \%$. En lo que corresponde al área urbana, para el año 2006 fue de un $8.8 \%$ y para el año 2011 de un $7.3 \%$. En el área rural se puede observar que en 2006 fue de un $20.9 \%$, ascendiendo para el año 2011 en un $22.0 \%$. Frente a este panorama es posible deducir que el área que se encuentra mayormente afectada es la rural, la cual presenta las tasas de analfabetismo más alta durante el período.

Según el gráfico 4, para el año 2006 la tasa de analfabetismo total presentó un $17.5 \%$ y para el año 2011 un $14.9 \%$, lo que refleja una disminución importante en el tema de educación. De forma similar se aprecia una disminución en el área urbana, ya que en 2006 se observó una tasa de analfabetismo de un 8.8 \% y para el año 2011 de un $7.3 \%$. En el caso del área rural la situación es menos alentadora, pues para el año 2006 se mostró un $20.9 \%$ y para 2011 una tasa del $22.0 \%$. En síntesis, se concluye que en el tema de educación en la parte rural es en donde mayores esfuerzos se necesitan realizar y es el área con mayor nivel de pobreza y analfabetismo que se encuentra en el país. Por lo que la tendencia en esta comparación se centra en el área rural, donde se espera que en vez de disminuir la tasa de analfabetismo se incremente. 
Gráfico 4. Tasa de analfabetismo según dominios, 2006 - 2011

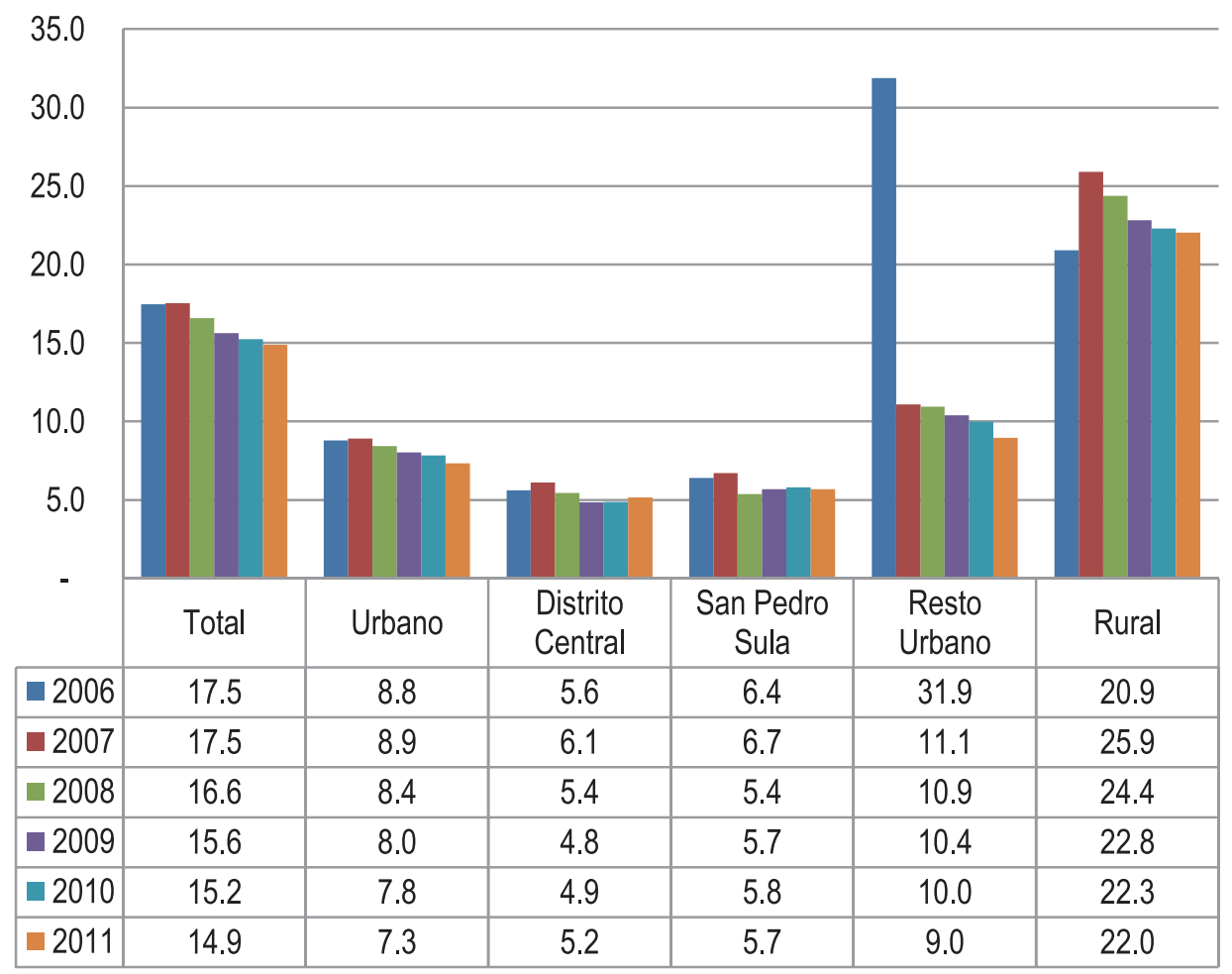

Fuente: elaboración propia con base a INE, EPHPM, mayo de 2006-2011.

2. Tendencia de la tasa de analfabetismo según edades en el período 2006-2011

Al observar la tendencia de la tasa de analfabetismo de acuerdo a las edades, esta indica que el problema se ha venido agudizado en un sector específico de la población, que es el de las personas de mayor edad. Así, se observa que para el año 2006 la tasa de analfabetismo alcanzó un $46.93 \%$ y en 2011 fue del $47.89 \%$.

En la población más joven, se observa una situación similar; por ejemplo, para el año 2006 presentó una tasa de analfabetismo del $5.86 \%$ y para el año 2011 de un $2.87 \%$. En consecuencia, es posible observar que en ambos casos se ha presentado una mejoría significativa en estos grupos de población (ver gráfico 5). 
Gráfico 5. Tasa de analfabetismo según edades, 2006 - 2011

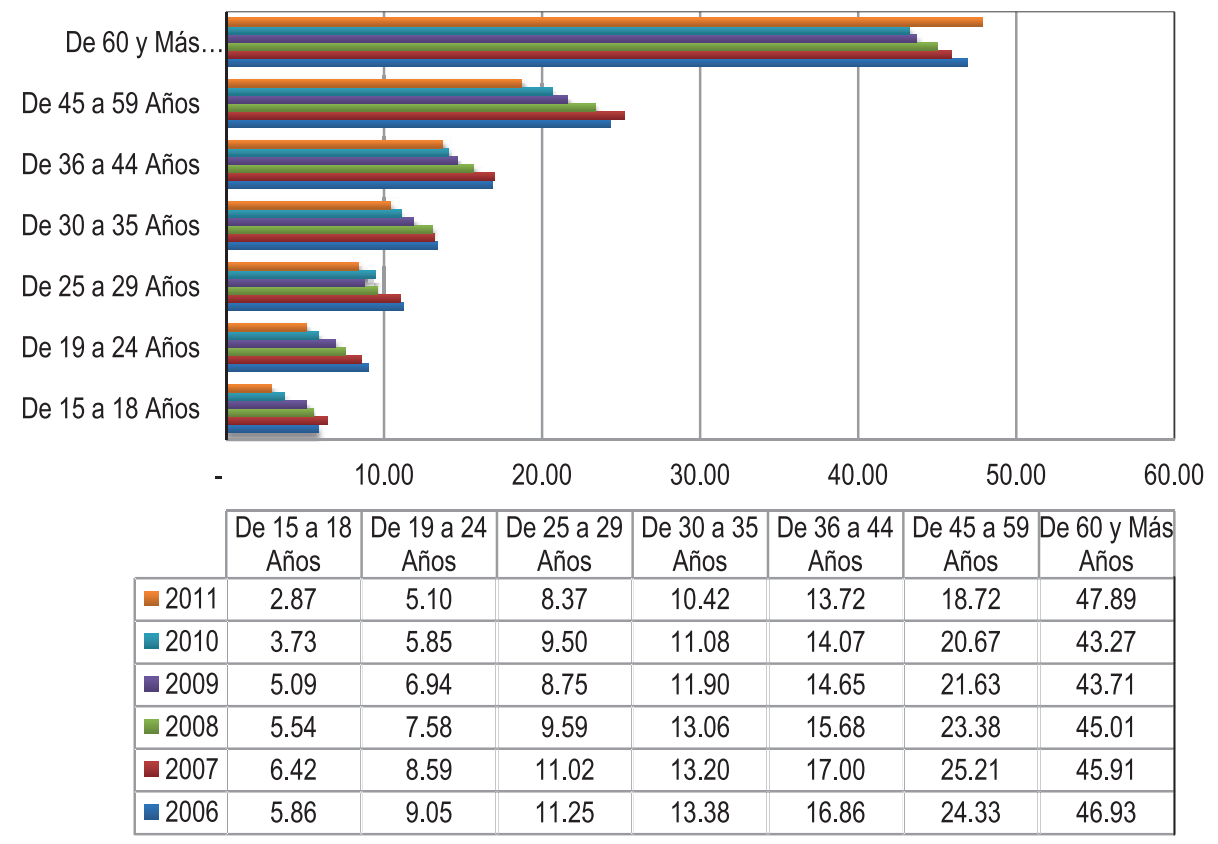

Fuente: elaboración propia con base a INE, EPHPM, mayo de 2006-2011.

El gráfico 5 muestra los grupos de edades en donde de todos los grupos el más afectado por el analfabetismo es la tercera edad, el cual para el año 2006 tuvo una tasa del $46.93 \%$ y para 2011 presentó un $47.89 \%$; con lo cual se convirtió en el único segmento de la población que incrementó este problema para el año 2011. De forma general se puede deducir que la tendencia de la tasa de analfabetismo tiende a disminuir de acuerdo a este comportamiento durante este período.

3. Caracterización del ingreso per cápita 2006-2011

El ingreso per cápita, estimado con base en los datos estadísticos del INE, ha sido estimado en el área rural y urbana, en miles de lempiras; en los cuales se aprecia una brecha. Así, para el año 2006 los ingresos per cápita en las áreas rural y urbana fue de L.1, 051.00 y de L. 2,755.00, respectivamente. Para el año 2011, los ingresos per cápita en las áreas rural y urbana ascendieron a L.1,916.00 y L.3,669.00, respectivamente.

Los resultados estimados en el país muestran que el área rural ha tenido un lento ascenso en el transcurso del período de estudio y el ingreso per cápita 
total en el país, para los años 2006 y 2011, fue de L.1, 887.00 y L. 2,774.00, respectivamente.

Lo que se puede deducir en cuanto el ingreso per cápita, es que hay una mejora en la posición de la población del área urbana, la cual presenta mayores ingreso con respecto al área del rural; en la primera los costos de la vida son más altos que la segunda.

Gráfico 6. Ingreso per cápita, urbano-rural, 2006 - 2011

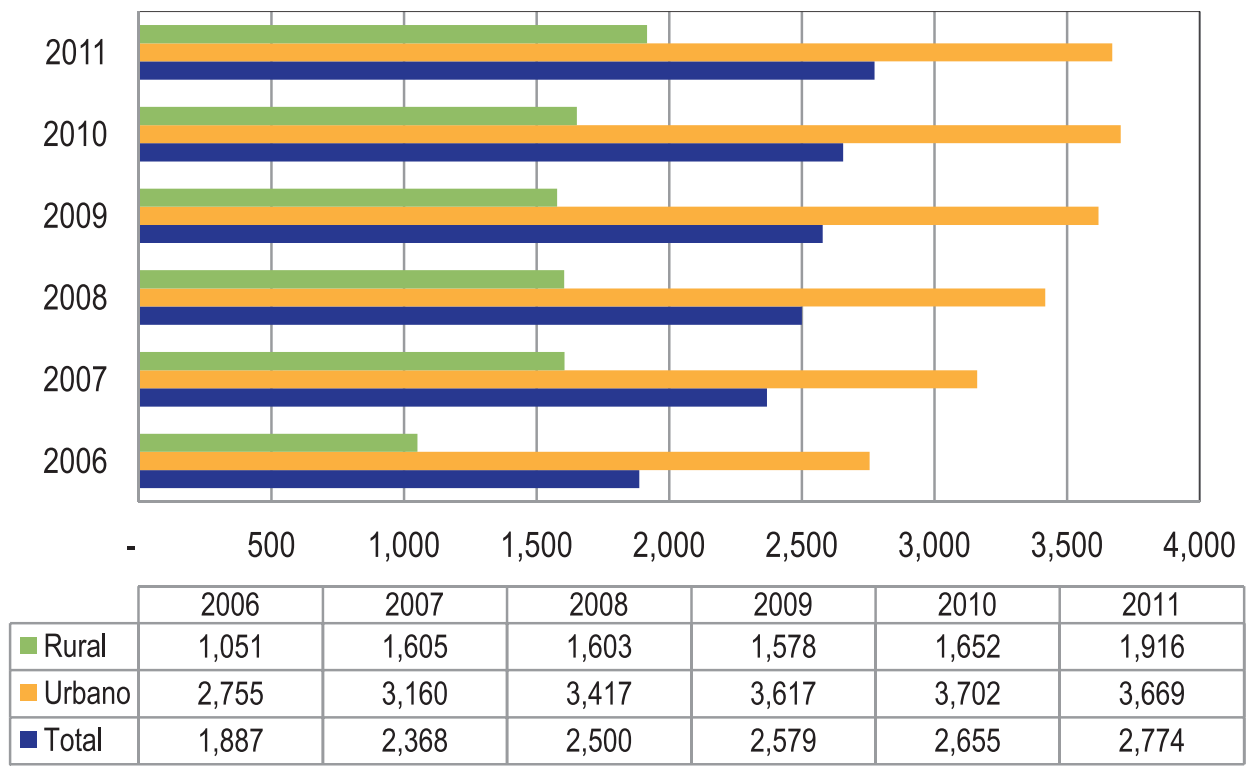

Fuente: elaboración propia con base a INE, EPHPM, mayo de 2006-2011.

En el gráfico 6 se presentan los ingresos per cápita que han venido ascendiendo durante el periodo estudio. En el sector urbano se muestra que para los años 2006 y 2011, los ingresos pasaron de L. 2,755.00 a L. 3,669.00, respectivamente; para el sector rural, de forma similar se presentaron incrementos, para 2006 fue de L.1, 051.00 y para el 2011 fue de L.1, 916.00, lo que refleja un incremento leve en cada uno de los años, en donde ambos sectores tienen factores por lo cuales estos incrementos no son suficientes para satisfacer las necesidades básicas de la población.

4. Tendencia del coeficiente $o$ índice de Gini en Honduras

Las expectativas pueden ser desalentadoras al analizar el planteamiento hecho por la el Informe de Desarrollo Humano Honduras 2011, el cual plantea 
que para acelerar la reducción de la pobreza es indispensable la disminución de la inequidad en Honduras.

Las medidas más conocidas para analizar la inequidad en la distribución de los ingresos es el coeficiente de Gini, el cual se expresa como un número ubicado entre 0 y 1 . El cero representa la perfecta igualdad (todos los habitantes del país tienen el mismo ingreso) y el uno la total inequidad (una persona tiene todo el ingreso y los demás no tienen nada).

Honduras tiene uno de los coeficientes de Gini más altos de América Latina, lo cual indica que es uno de los países con mayor inequidad en la distribución de los ingresos en la región. Si se observa el período de estudio, el coeficiente Gini para el año 2006 se mostró en 0.596; para el año 2009 fue de 0.525 y para el año 2011 se registró un 0.580 , lo cual indica el nivel de la distribución de los ingresos, que en comparación con la región es uno de los países con mayor inequidad en la distribución de los ingresos en la región, quedando evidenciado que Honduras supera a los demás países del istmo centroamericano IDH (2011).

Gráfico 7. Coeficiente o índice Gini, 2001 - 2011

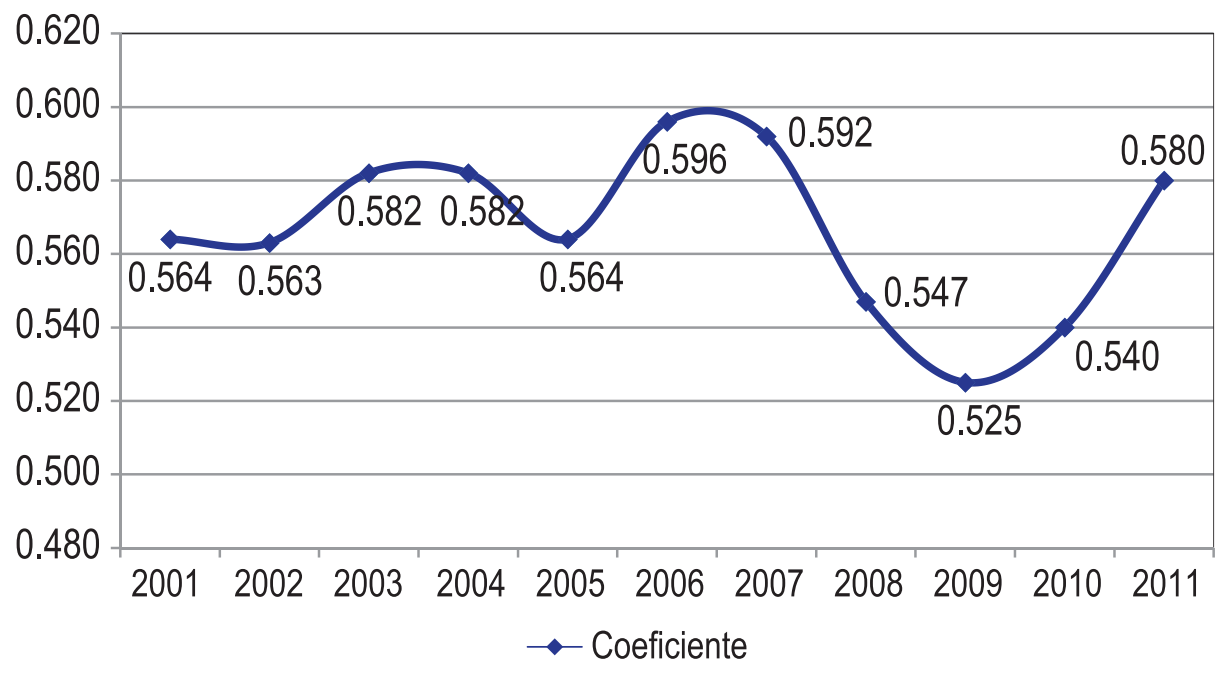

Fuente: elaboración propia con base al IDH, 2011. 
5. Análisis econométricos de la pobreza, el analfabetismo y el índice de Gini, en el período 2001-2011

Para explicar la relación de la pobreza con el analfabetismo y el índice de Gini, es necesario conocer y estudiar a profundidad las causas de la pobreza, ya que la educación y la distribución del ingreso actúan como factores cooperantes al mejoramiento de los niveles estándares de la calidad de vida, contribuyendo con ello a las disminución de la precariedad humana.

En este caso, se modelará la relación de la pobreza entre el analfabetismo y el índice de Gini, según se expone en este modelo propuesto, de forma que si aumentan los niveles de educación en el país (ceteris paribus), los niveles de pobreza tenderían a disminuir y de igual forma con un aumento de la desigualdad del ingreso, representado con una disminución del índice Gini, se esperaría una disminución de la pobreza.

D. Hipótesis del modelo

- H: 1 Con el aumento de los niveles de educación (disminución de la tasa de analfabetismo) los niveles de pobreza disminuyen.

- H: 2 Con la disminución del índice Gini (mejor distribución del ingreso) existe una disminución de los niveles de pobreza.

E. Estimación econométrica

Después de describir las variables que explican la relación existente entre el analfabetismo y la distribución del ingreso, se presentan las estimaciones econométricas que permiten establecer relaciones.

Se ha definido la pobreza en función de la tasa de analfabetismo y la distribución del ingreso medido a través del índice de Gini:

Pobreza $=F($ analfabetismo, índice de Gini)

Donde se ha realizado una contrastación empírica del modelo que se ha planteado: $Y=\beta 1+\beta 2$

A través del análisis econométrico en la aplicación de las herramientas estadísticas mediante el programa stata, se realizó la regresión y el análisis de los resultados con la finalidad de dotar las relaciones entre las variables. 
Básicamente se trata de conocer las relaciones básicas entre variables de interés (pobreza y analfabetismo y el índice de Gini).

El modelo de regresión: $\mathrm{LnP}=\mathrm{At}+\ln$

Donde P: pobreza total; At: analfabetismo; In: índice de Gini

Tabla 1. Resultado de la estimación del modelo

. reg logPOBREZA_TOTAL resTOTAL_ANAL resGINI

\begin{tabular}{|c|c|c|c|c|c|c|}
\hline Source & ss & df & ms & & Number of obs $=$ & 8 \\
\hline Model & 0.076857999 & 2 & 0.038428999 & & $\begin{array}{l}f(2,5)= \\
\text { Prob }>F=\end{array}$ & $\begin{array}{r}10.13 \\
0.0174\end{array}$ \\
\hline Residual & 0.01896314 & 5 & 0.003792628 & & $\begin{array}{l}R \text { - squared }= \\
\text { Adj } R \text {-squared }=\end{array}$ & $\begin{array}{l}0.8021 \\
0.7229\end{array}$ \\
\hline Total & 0.095821139 & 7 & 0.013688734 & & RootMSE = & 0.06158 \\
\hline logPOBREZA_ L & Coef. & Std. Err. & $\mathrm{t}$ & $\mathrm{p}>|\mathrm{t}|$ & \multicolumn{2}{|c|}{ ( $95 \%$ Conf. Interval) } \\
\hline resTOTAL_ANA & $7.20 \mathrm{E}-06$ & $1.60 e-06$ & 4.48 & 0.006 & $3.07 e-06$ & 0.0000113 \\
\hline resGINI & -4.006115 & 1.583491 & -2.53 & 0.053 & -8.076608 & 0.0643782 \\
\hline cons & 12.02599 & 1.136712 & 10.58 & 0.000 & 9.103983 & 14.948 \\
\hline
\end{tabular}

end of do - file

Fuente: elaboración propia con base a la regresión estimada con stata.

Después de haber realizado las estimaciones en la tabla 1, se observa como el modelo econométrico, con un nivel de significancia del $95 \%$, muestra que la variable analfabetismo influye de forma positiva en el nivel de la pobreza; a medida que se incrementa el número de personas analfabetas, el nivel de pobreza es mayor; con esta estimación se comprueba que con el aumento de los niveles de educación hay una disminución de la tasa de analfabetismo (hipótesis 1) y los niveles de pobreza disminuyen. Lo que significa que hay una la relación directa en la educación para el desarrollo, lo cual es pilar fundamental en una sociedad para el incremento de su calidad de vida. Con esta relación se perciben puntos claves de la importancia que posee la educación para la sociedad y el mejoramiento en la calidad de vida, con la disminución de los niveles de pobreza en el país.

Con respecto a la segunda, la variable Gini a nivel de significancia es del $95 \%$, lo que representa una relación inversa, que para efectos de lo que se entiende por la lógica Gini, es que mientras más cercano se encuentra este índice a la unidad, existirá un mayor grado de desigualdad en los ingresos, por lo que en este caso se ha encontrado una relación espuria, lo cual indica algo irracional, cuyo 
significado es que si se incrementa el índice Gini (mayor grado de desigualdad), los niveles de pobreza disminuyen; por lo tanto, en esta regresión no se acepta la hipótesis 2: con la disminución del índice Gini (mejor distribución del ingreso), existe una disminución de los niveles de pobreza; que es exactamente lo que se busca sustentar con el estudio de la relación del ingreso per cápita y los niveles de pobreza.

En forma general, el nivel de pobreza de la población del país se encuentra relacionado de forma positiva con el analfabetismo, pero también se espera que al mostrar mayores niveles de distribución del ingreso se disminuya la cantidad de personas en condición de pobreza, lo cual con la regresión no ha sido posible constatar.

\section{CONCLUSIONES}

1. No queda duda de que la pobreza en Honduras se ha originado históricamente. Aunque explorar este tema en un pequeño espacio de tiempo no es muy recomendable, se puede considerar positivamente que este estudio aportó a la caracterización de aspectos fundamentales para el comportamiento y entendimiento de la pobreza. Al respecto, al observar de forma gráfica la tendencia de los niveles de la pobreza, las tasas de analfabetismo y los ingresos per cápita, se ve que estos contribuyeron de forma moderada al propósito del estudio, en cuanto a la explicación de los resultados de la pobreza.

2. En definitiva, el tema de la pobreza sigue siendo materia de estudio, aunque con un difícil camino hacia su erradicación. Las causas de la pobreza se atribuyen a un problema estructural y a las crisis económicas-financieras a nivel nacional e internacional, las cuales generan desequilibrios en los distintos indicadores económicos cuyos efectos se pueden medir por problemas en la inversión y, por ende, en el desempleo, lo que está generando falta de riqueza. De esto depende la distribución y el mecanismo de distribución, con lo que la variable ingreso ejerce una potente influencia al momento de atacar parte del problema, pero no es lo suficiente para resolver el flagelo de la pobreza; por eso es necesario avanzar de forma importante en el capital social. En esencia, el punto es más complejo es que se pueden atacar los aspectos económicos de la economía nacional, pero en el fondo no se está resolviendo el punto central de la pobreza.

3. La tendencia de los niveles de pobreza en los próximos años es hacia el incremento, lo que ya es trágico para el país, pero sería lamentable no buscar alternativas que detengan esta tendencia, lo que vendría a profundizar la 
indigencia y los principales problemas sociales del país, tales como la violencia, inseguridad, narcotráfico, crimen organizado, etc.

4. La población en condición de pobreza que esta siendo más afectada por este flagelo, es la que está en la área rural. Esta presenta una situación mucho más crítica que el resto de la población, dado que se encuentra desprotegida del bienestar económico y social, carece de alternativas de desarrollo y sufre de forma directa los abates de este fenómeno social; en consecuencia, es posible afirmar que los programas que se han implementado, como la estrategía de la reducción para la pobreza, no han sido suficientes y en ese sentido el Gobierno deberá fortalecer o generar alternativas para que estos programas aporten mejoras en este tema.

5. La pobreza en Honduras, sobre toda la pobreza extrema, que es la que demuestra que la situación es mucho más severa,con respecto a la pobreza relativa, que muestra niveles considerables, por otro lado, a través de mayores niveles de educación en los sectores de la población mayor afectada, sobre todo en el área rural donde se encuentran en bajos niveles de educación y formación.

6. La educación en Honduras presenta tendencias que son importantes de considerar. Por lo que se observa en este período de estudio, lo que se destaca es la tendencia hacia la disminución de la tasa de analfabetismo; en tal sentido, la población hondureña puede erradicar este problema, pero para ello se deberá continuar fortaleciendo de mejor forma el enfasís en el área rural, que sigue siendo la más afectada y, sobre todo, al segmento de la población que corresponde a la tercera edad.

7. Con respecto al ingreso per cápita de las áreas rural-urbana, las personas que se encuentran en el área rural son las que menos ingresos en promedio reciben, de manera que es el segmento que con mayor fuerza afecta la pobreza. Con esto se puede deducir que este segmento muestra un bajo ingreso y una tasa de analfabetismo alta. Consecuentemente, la salida al tema de la pobreza pasa por el aporte que pueden brindar los niveles de educación y el desarrollo que pueda generarse en las zonas de producción agrícolas.

8. El ingreso per cápita en Honduras está marcado por una brecha entre el urbano y lo rural, con lo cual queda demostrado que los ingresos en promedio en el área urbana son totalmente superiores a los del área rural. De igual manera, en lo que respecta a la educación, predominan mejores niveles en el área urbana con respecto al área rural, lo que demuestra que este es un factor determinante en 
los niveles de ingresos en la población y que a mejores niveles de ingreso, mejor es la condicion de vida de la población.

9. En conclusión, se puede decir que la pobreza es un fenómeno que puede ser reducido de forma significativa con el mejoramiento y la continuidad de progamas de educación, los que han estado aumentando los niveles de educación en la sociedad hondureña. De acuerdo a los resultados, este tiene un efecto importante en los ingresos de la población, lo que puede explicar, en cierta forma, la estacionalidad o la reducción de la pobreza para este período de estudio; así, queda claro que la pobreza no solo puede ser explicada por estas variables, ya que existen otros enfoques que también la explican quizas de mejor forma.

\section{BIBLIOGRAFÍA}

Banco Mundial. (2006). Honduras, reporte de pobreza. Logrando la reducción de la pobreza. Recuperado de: http://www.youblisher.com/p/365637-HONDURASREPORTE-DE-POBREZA-Logrando-la-reduccion-de-la-pobreza/

BCIE. Ficha estadística de Honduras. Recuperado de: http://www.bcie.org/uploaded/content/article/1944368211.pdf

Caseros Cañas, Ana. Estudio pobreza. Recuperado de: ftp://ftp.fao.org/TC/TCA/ESP/pdf/casero/Bloquel.2.pdf

CEPAL. (2008). Superar la pobreza mediante la inclusión social. Recuperado de: www.eclac.cl/id.asp?id=32358

PNUD. (2012). Estrategia para la reducción de la pobreza. Recuperado de: http://www.undp.un.hn/documentos/ERPCompleto.pdf

Feres, Juan Carlos y Mancero, Xavier. Enfoques para la medición de la pobreza. Breve revisión literaria. Recuperado de: http://www.eclac.cl/deype/mecovi/docs/TALLER4/5.pdf

Instituto Nacional de Estadística y Censos de Costa Rica. Medición de la pobreza, método de la línea de pobreza. Recuperado de: http://www.eclac.cl/deype/mecovi/docs/TALLER13/11.pdf

Jiménez Castillo, Juan. (2007). La investigación sobre el analfabetismo funcional. Estado actual del concepto. Recuperado de: web.usal.es/ efora

Mena, Roberto. (2008). Pobreza y educación. Recuperado de: www.ues.edu.sv/INVE

Mendoza León, Juan. Educación y nivel de ingreso departamental en el Perú. Recuperado de:

sisbib.unmsm.edu.pe/bibvirtualdata/.../16/.../educacion_ingreso.pdf

ODM1. Erradicar la pobreza extrema y el hambre. Recuperado de: www.undp.un.hn/ODM2010/InformeODM2010/ODM1.pdf 
Patnaik, Utsa. (2010). Teorías sobre la pobreza y la seguridad alimentaria en la era de las reformas económicas. Recuperado de: http://seguridadysoberaniaalimentaria.org

PNUD. (2012). Informe Sobre Desarrollo Humano Honduras 2011. Reducir la inequidad: un desafío impostergable. Costa Rica.

Revallion, Martin. Las líneas de pobreza en la teoría y en la práctica. Recuperado de: www.austral.edu.ar/aplic/webSIA/webSIA2004.nsf/.../Ravallion.pdf

Rodríguez Ramírez, Héctor. Enfoques para la medición de la pobreza: breve revisión de la literatura. Recuperado de:

http://www.mty.itesm.mx/egap/centros/caep/imagenes/Pobreza.pdf

Verdera V., Francisco. Enfoques de pobreza. Recuperado de:

http://biblioteca.clacso.edu.ar/ar/libros/coedicion/verdera/02enfoque.pdf

Villa, Manuel y Lovo, Horacio. Crisis y pobreza rural en Honduras. Recuperado de:

http://www.rimisp.org/FCKeditor/UserFiles/File/documentos/docs/pdf/DTR/crisis/ Crisis-pobreza-rural-Honduras-policy-briefs-DTR.pdf 